\title{
Exercise Effects on Erythrocyte Deformability in Exercise-induced Arterial Hypoxemia
}

\author{
Authors \\ R. Alis ${ }^{1,5}$, F. Sanchis-Gomar ${ }^{1,2,3}$, D. Ferioli ${ }^{4}$, A. La. Torre ${ }^{4}$, J. R. Blesa ${ }^{1,5}$, M. Romagnoli ${ }^{1,6}$ \\ Affiliations \\ Affiliation addresses are listed at the end of the article
}

\author{
Key words \\ - exercise \\ - exercise-induced arterial \\ hypoxemia \\ - erythrocyte deformability
}

accepted after revision September 10, 2014

\section{Bibliography}

DOI http://dx.doi.org/

10.1055/s-0034-1394395

Published online: 2014

Int J Sports Med

(c) Georg Thieme

Verlag KG Stuttgart · New York

ISSN 0172-4622

\section{Correspondence}

\section{Rafael Alis}

Research Universitary Institute

Dr. Viña Giner, Molecular and

Mitochondrial Medicine,

Catholic University of Valencia

San Vicente Mártir

C/Quevedo 2

Valencia 46001

Spain

Tel.: + 34/963/637412

Fax: + 34/963/637 412

rafael.alis@ucv.es

\section{Abstract \\ $\nabla$}

Exercise-induced arterial hypoxemia (EIAH) is often found in endurance-trained subjects at high exercise intensity. The role of erythrocyte deformability (ED) in EIAH has been scarcely explored. We aimed to explore the role of erythrocyte properties and lactate accumulation in the response of ED in EIAH. ED was determined in 10 sedentary and in 16 trained subjects, both before and after a maximal incremental test, and after recovery, along with mean corpuscular volume (MCV) and red blood cell lactate concentrations. EIAH was found in 6 trained subjects $\left(\Delta \mathrm{SaO}_{2}=-8.25 \pm 4.03 \%\right)$. Sedentary and nonEIAH trained subjects showed reduced ED after

\section{Introduction}

$\nabla$

During incremental exercise, a drop in arterial $\mathrm{O}_{2}$ saturation $\left(\mathrm{SaO}_{2}\right)$ is frequently observed in male endurance-trained subjects [19]. This effect, named exercise-induced arterial hipoxemia (EIAH), is believed to reflect an insufficient alveolar gas exchange, probably caused by mismatch in alveolar ventilation to perfusion ratio and diffusion limitations [21]. A rheological parameter, blood viscosity is a function of plasma viscosity, red blood cell (RBC) aggregation, hematocrit and erythrocyte deformability (ED), with ED being the main determinant in microcirculation [4]. Several published observations suggest a role of blood rheological properties in EIAH development: i) blood viscosity rise induces pulmonary vascular resistance increment [20]; ii) an artificially induced decrease in ED diminishes lung $\mathrm{O}_{2}$ diffusion capacity in animal models [6]; iii) administering polyunsaturated fatty acids to EIAH subjects, which improves ED, reduces EIAH severity during incremental exercise $[1,2]$. However, the blood rheology response to exercise in EAIH has been scarcely explored. It have exercise, while no effect on ED was found in EIAH trained subjects. After exercise, lactate concentrations rose and MCV increased equally in all groups. ED is strongly driven by cell volume, but the different ED response to exercise in EIAH shows that other cellular mechanisms may be implicated. Interactions between membrane and cytoskeleton, which have been found to be $\mathrm{O}_{2}$-regulated, play a role in ED. The drop in $\mathrm{SaO}_{2}$ in EIAH subjects can improve ED response to exercise. This can be an adaptive mechanism that enhances muscular and pulmonary perfusion, and allows the achievement of high exercise intensity in EIAH despite lower $\mathrm{O}_{2}$ arterial transport.

been shown that during incremental exercise, blood viscosity increased substantially more in EIAH than in non-EIAH subjects, which may be due to different cellular properties [11]. In fact, increased ED [11], decreased ED [13] or absence of effects on ED [8] have been reported in EIAH, despite most of the published studies having shown that ED decreases with exercise [10].

It has been proposed that lactate accumulation may play a pivotal role in affecting ED during exercise [10], since in vitro, it exerts opposite effects on ED in trained and non-trained subjects [9]. Interestingly in highly trained subjects, those showing EIAH display greater lactate influx activity, mediated by monocarboxylate transporter-1 (MCT-1) [8].

The results for ED in EIAH are controversial and can be attributed to methodological issues such as exercise modality [13] or use of indirect ED measurement [31]. However, the role of ED in EIAH appearance warrants further research.

In the present study, we aimed to ascertain by ektacytometry techniques whether ED response to exercise differs between EIAH and non-EIAH subjects. We also aimed to explore whether RBC 
lactate uptake during exercise plays a role in ED between EIAH and non-EIAH subjects. For this purpose, trained and nontrained subjects performed a running incremental test until exhaustion, with $\mathrm{SaO}_{2}$ being monitored. ED, plasma and RBC lactate concentrations were measured before and after exercise, and after a 30-min recovery period.

\section{Materials and Methods}

$\nabla$

\section{Subjects}

Thirty healthy male subjects were enrolled in the study. 20 were physically active (trained group, triathletes and endurance athletes who pursued physical activity weekly $>12 \mathrm{~h}$ ) and 10 subjects were physically inactive (sedentary group, weekly physical activity $<1 \mathrm{~h}$ ). Exclusion criteria were hematological, infectious or inflammatory diseases, history of heart disease, hormonal impairments (i.e., hypo- or hyperthyroidism) or presence of cardiovascular risk factors (obesity $\left(\mathrm{BMI}>30 \mathrm{~kg} / \mathrm{m}^{2}\right)$, current tobacco use ( $>1$ cigarette/day), hypertension (systolic blood pressure $>140 \mathrm{mmHg}$, diastolic blood pressure $>90 \mathrm{mmHg}$ ), hyperlipidemia (total cholesterol $>220 \mathrm{mg} / \mathrm{dL}$ and/or triglycerides $>175 \mathrm{mg} / \mathrm{dL}$ ) or fasting glucose $(>126 \mathrm{mg} / \mathrm{dL})$ ). 4 subjects (all belonging to the trained group) were excluded since they did not complete the incremental test or it was considered not maximal. Therefore, 26 healthy male (10 sedentary and 16 trained) subjects were ultimately included in the study ( $\bullet$ Table 1 ).

All of the participants were informed of the purpose, protocol and procedures before agreeing to participate in the study, which was approved by the Ethical Committee of the Catholic University of Valencia. This work complies with the principles of the Declaration of Helsinki and was performed in accordance with the Spanish laws on research in humans. This study meets the ethical standards of the International Journal of Sports Medicine [14].

\section{Experimental protocol}

All participants reported to the laboratory in fasting conditions between 8 and 9 a.m. Subjects performed a maximal incremental test on a motorized treadmill until exhaustion. During the warm-up period $(5 \mathrm{~min})$ subjects selected the running speed (between 8 and $12 \mathrm{~km} / \mathrm{h}$ ) according to their preferences and habitual training pace. This speed remained fixed during the test, while the slope was increased by $1 \%$ every minute. The test ended when the subject was unable to keep running despite verbal encouragement. Ventilatory parameters were recorded during exercise through a respiratory valve and a face mask (Hans Rudolph, Inc., Kansas City, MO, USA) using a gas analyzer (MetaLyzer 3B-R2, Cortex GmbH, Germany). The test was considered maximal when at least 2 of the following conditions were fulfilled: respiratory quotient $>1.1$ and maximal heart rate $>$ maximal heart rate predicted as 220 -age.

During the incremental test, $\mathrm{SaO}_{2}$ was non-invasively recorded every 15 s by pulse-oximetry using a Cardell 9500 HD Multiparameter Monitor. Presence of EIAH was considered when $\mathrm{SaO}_{2}$ decreased by at least $4 \%\left(\Delta \mathrm{SaO}_{2} \leq-4 \%\right)$ from the baseline values in the last 2 min of the incremental test [21].

\section{Blood sampling}

Blood samples were taken from the antecubital vein before, just after exercise and after the 30-min recovery period. Blood was collected in standard Vacutainer ${ }^{\circledR}$ tubes containing $\mathrm{K}_{3}$ EDTA for lactate, hematological and hemorheological measurements. Tubes were kept in ice until performing determinations within 2-h after extraction.

\section{Laboratory methods}

Erythrocyte deformability was determined by ektacytometry techniques in a Rheodyn shear stress diffractometer (Myrenne $\mathrm{GmbH}$, Germany) at $60 \mathrm{~Pa}[23,25]$. In short, $30 \mu \mathrm{l}$ of blood were suspended in $2 \mathrm{ml}$ of a dextran solution (24\% dextran 40000 ; $\mathrm{pH}=7.4,300-310 \mathrm{mOsm} / \mathrm{L}$ and $24 \mathrm{mPa} \cdot \mathrm{s}$ viscosity). The blooddextran suspension was placed between a rotating optical glass disc and a stationary disc. A defined shear force of 0.3-60 Pa was exerted on this suspension. Red blood cells were aligned with the fluid shear forces in parallel to the direction of flow and deformed to ellipsoids. A helium-neon laser beam passed through the red blood cell suspension and a diffraction pattern appeared. The diffraction pattern is circular with red blood cells at rest, but becomes elliptical when cells are deformed by shear. The light intensities of the diffraction pattern were measured along 2 orthogonal axes equidistant from the center of the image, and the erythrocyte elongation index (EEI) was calculated from the width (W) and length (L) of the ellipsoid:

Table 1 Subjects characteristics and incremental test data comparison between the sedentary subjects and trained subjects who did not present exerciseinduced arterial hypoxemia (EIAH) and those who did.

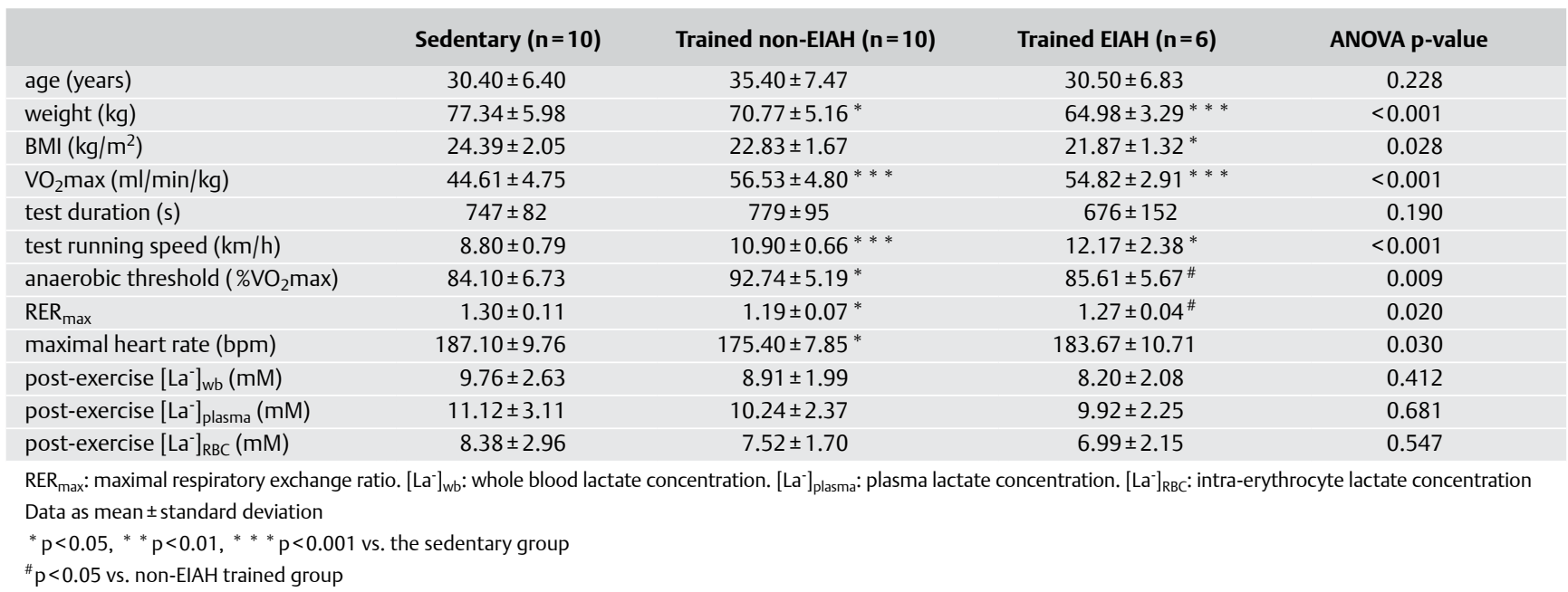


$\mathrm{EEI}=100 \cdot(\mathrm{L}-\mathrm{W}) /(\mathrm{L}+\mathrm{W})$. We determined EEI at $60 \mathrm{~Pa}(\mathrm{EEI} 60)$ in duplicate in all of the samples. The inter-assay variation coefficient of the technique was $0.98 \%$.

Hematocrit (Htc), hemoglobin, RBC and reticulocyte counts, and cellular indices - i.e., mean corpuscular volume (MCV), mean corpuscular hemoglobin $(\mathrm{MCH})$ and mean corpuscular hemoglobin concentration (MCHC) - were determined in a Sysmex XE-2100L (Sysmex, Kobe, Japan).

The lactate concentrations in whole blood and plasma were determined just after drawing with a Biosen C-line autoanalyzer (EKF-diagnostic GmbH, Germany). After blood was drawn, two $120 \mu \mathrm{l}$ pre-cooled capillary tubes were quickly filled with anticoagulated blood and centrifuged for $30 \mathrm{~s}$ at $21000 \mathrm{~g}$ and $4^{\circ} \mathrm{C}$ (Sorvall Legend Micro 21R Microcentrifuge, Thermo Scientific, Germany). Afterwards, plasma was separated from the pelleted RBCs and used for the lactate measurements. Then $20 \mu \mathrm{l}$ of either whole blood or plasma were pipetted into the pre-made analyzer tubes, gently agitated and inserted into the previously calibrated analyzer. All of the measures were taken in duplicate. Intra and inter-assay variation coefficientes were $0.92 \%$ and 2.39 $\%$ for the whole blood lactate measures, and $0.73 \%$ and $1.21 \%$ for the plasma lactate measures. Intra-erythrocyte lactate concentrations were estimated using the following equation [15]:

$\left[\mathrm{La}^{-}\right]_{\mathrm{RBC}}=\left[\left(100 \cdot\left[\mathrm{La}^{-}\right]_{\mathrm{WB}}\right)-\left((100-\mathrm{Hct}) \cdot\left[\mathrm{La}^{-}\right]_{\text {Plasma }}\right)\right] \cdot \mathrm{Htc}^{-1}$

where $\left[\mathrm{La}^{-}\right]_{\mathrm{RBC}}$ is the lactate concentration inside the erythrocyte, $\cdot\left[\mathrm{La}^{-}\right]_{\mathrm{WB}}$ is the whole blood lactate concentration and $\left[\mathrm{La}^{-}\right]_{\text {Plasma }}$ is the plasma lactate concentration.

\section{Statistical analysis}

All of the data variables were analyzed for normality by the Shapiro-Wilk test. Whenever required, data were log-transformed to improve normality for statistical testing. The statistical analyses were performed by grouping subjects according to their training status and presence of EIAH (sedentary, trained nonEIAH and trained EIAH). Incremental test data, subjects' characteristics and the hematological parameters were analyzed in a one-way ANOVA for independent measures. Homogeneity of variance was assessed by Levene's test. When this assumption was violated, Welch's correction was adopted. Exercise effects on ED, erythrocyte indices and $\mathrm{La}^{-}$concentrations, were analyzed using a one-way ANOVA for repeated measures (sampling time: pre-exercise, post-exercise and 30-min recovery). Post hoc comparisons were made with Bonferroni's correction. Pearson's correlation coefficients were calculated in order to explore the association between continuous variables. The statistical analysis was performed using SPSS, version 21 (IBM Corporation, Armonk, NY, USA). The results were considered statistically significant at $\mathrm{p}<0.05$. Data were expressed as mean \pm standard deviation (SD).

\section{Results}

$\nabla$

\section{Subjects characteristics}

In the last $2 \mathrm{~min}$ of the incremental test, 6 trained subjects showed arterial oxygen saturation at least $4 \%$ lower than the baseline levels $\left(\Delta \mathrm{SaO}_{2}=-8.25 \pm 4.03 \%\right.$ ). For the data analysis, trained subjects were therefore divided into those not showing EIAH and those showing EIAH. Sedentary subjects were heavier, their selected running pace was lower, and their $\mathrm{VO}_{2} \max$ was lower than in both trained groups ( $\bullet$ Table 1 ). Interestingly, trained EIAH subjects showed a higher maximal respiratory exchange ratio $\left(R E R_{\max }\right)$, and their anaerobic threshold was at a lower $\mathrm{VO}_{2}$ max percentage than for the non-EIAH trained subjects ( $\odot$ Table 1$)$. The mean EEI60 values were no different among the 3 groups $(F(2,25)=0.225 ; p=0.800)$. Moreover, no differences were found for the hematological parameters (Suppl. Table 1).

\section{Exercise effects on ED}

We analyzed the effects of exercise on ED, along with MCV and $\mathrm{MCHC}$, since these erythrocyte indices are major determinants of EEI60. Exercise lowered ED accompanied by cell swelling and, accordingly, decreased MCHC in the sedentary and trained nonEIAH subjects ( $\odot$ Fig. 1). However, in the trained subjects with EIAH, identical exercise-induced effects were observed on MCV and MCHC, but no effects were noted on EEI60 ( $\odot$ Fig. 1).

Correlation analysis was applied to further investigate the association between the exercise effects on ED and the presence of EIAH and cell swelling ( $\bullet$ Fig. 2). A significant inverse bivariate correlation was found between the exercise-induced change in EEI60 and the arterial $\mathrm{O}_{2}$ desaturation at the final $2 \mathrm{~min}$ of the incremental test $(r=-0.626, p<0.001, n=26)$. Interestingly, exercise-induced MCV increment was found to be correlated with the exercise effects on ED only when EIAH subjects were removed from analysis $(\mathrm{r}=-0.503, \mathrm{p}=0.024, \mathrm{n}=20)$ (Suppl. Table 2).

\section{Exercise effects on plasma and intra-erythrocyte lactate concentrations}

We aimed to test the hypothesis that lactate intake plays a role in the ED response to intense exercise in trained-EIAH subjects. Therefore, we measured plasma and whole blood lactate concentrations, and calculated the inside-to-outside RBC lactate ratio before and after exercise, and also after the 30-min recovery period. As expected, the lactate concentrations and the inside-to-outside RBC lactate ratio rose considerably after exercise ( $\odot$ Fig. 3) in all of the groups. However, no significant differences between groups were found in any lactate-related variable explored $(p \geq 0.346)$.

\section{Discussion \\ $\nabla$}

To accomplish their biological function RBCs must undergo major deformations in micro and macro circulation. ED is driven by the interplay of 3 parameters: membrane-cytoskeleton flexibility, surface-to-volume ratio and cytoplasmic viscosity. We demonstrate that intense exercise induces an increment in MCV, and a subsequent decrease in MCHC, irrespectively of training status and presence of EIAH. These effects should have a divergent impact on ED. As a result of cell swelling, the surface-tovolume ratio decreased, impairing ED, while cytoplasmic viscosity (directly related to hemoglobin concentration) also dropped, thus improving ED. However, within normal ranges, the effect of MCHC on ED is limited [12]. Therefore, ED should decrease as a result of these factors interaction. Indeed, the EEI60 data showed that exercise decreased ED in the sedentary and non-EIAH trained subjects ( $\bullet$ Fig. 1), but this effect was not observed in the trained EIAH subjects. Moreover, correlation data shows that EIAH appearance distorts the relationship between the exercise-induced cell swelling and ED impairing (० Fig. 2) - a finding that has been previously reported [22]. 

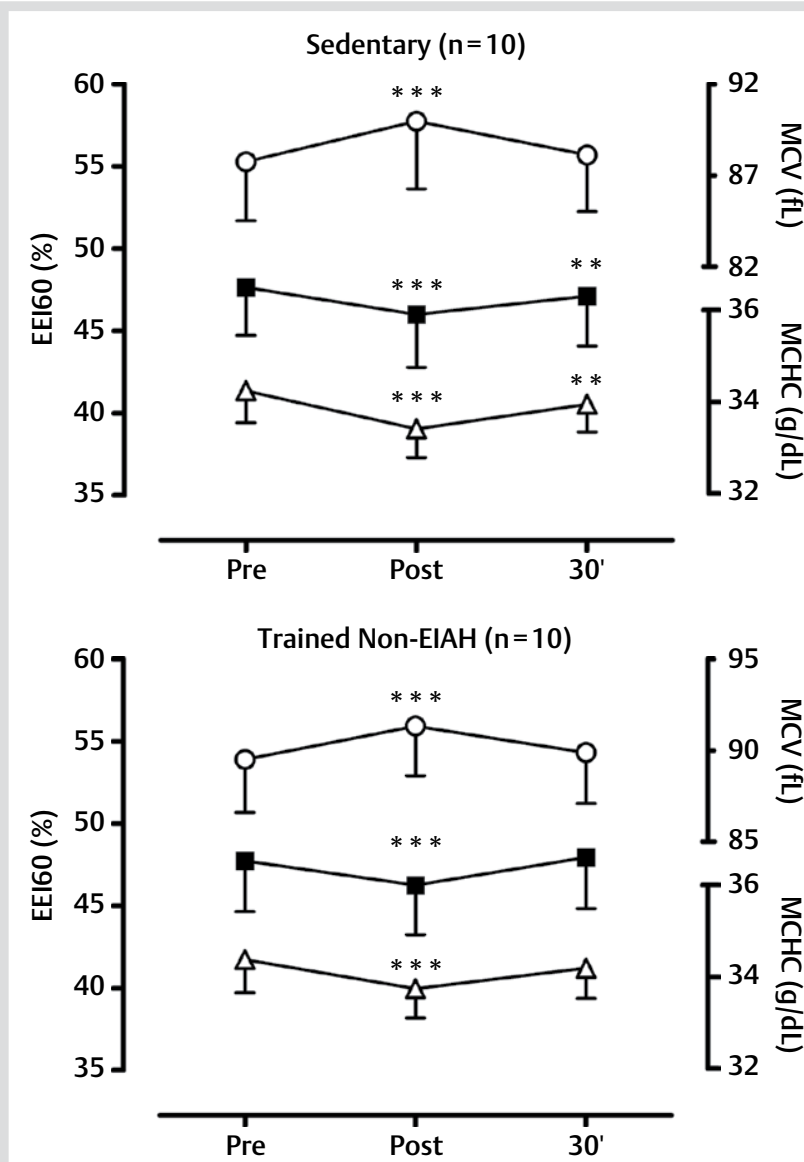

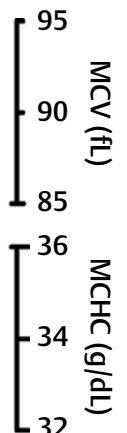

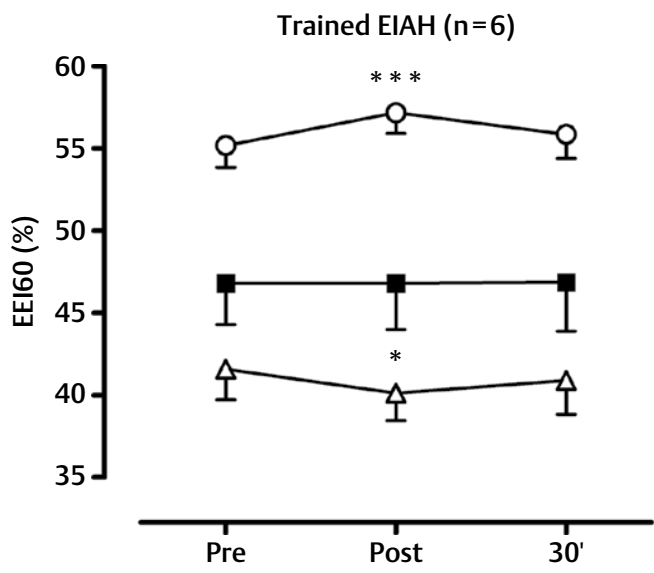

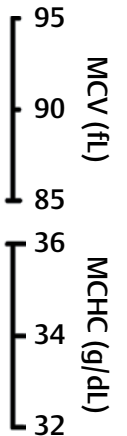

Fig. 1 Erythrocyte deformability (EEI60, 匹), erythrocyte mean corpuscular volume (MCV, $\bigcirc$ ) and erythrocyte mean corpuscular hemoglobin concentration (MCHC, $\triangle$ ) evolution during exercise and 30-min recovery, in the sedentary, the trained subjects without exercise-induced arterial hypoxemia (EIAH) and the trained subjects with EIAH. Symbols represent the mean values and error bars denote standard deviation. ${ }^{*}{ }^{*} \mathrm{p}<0.01,{ }^{* * *} \mathrm{p}<0.001$ vs. pre-exercise.

To the best of our knowledge, this is the first work to determine ED in EIAH directly by ektacytometry. Previous work that studied exercise effects on ED in EIAH have reported increased [11], decreased [13] or no changes [8] in ED. However, indirect ED measures (i.e., Tk calculations), which are currently outdated $[5,31]$, were employed $[8,11,13]$.

It has been previously shown that lactate uptake during exercise causes RBC swelling and decreased cell density [26], which is in agreement with the results of the present study. The trained

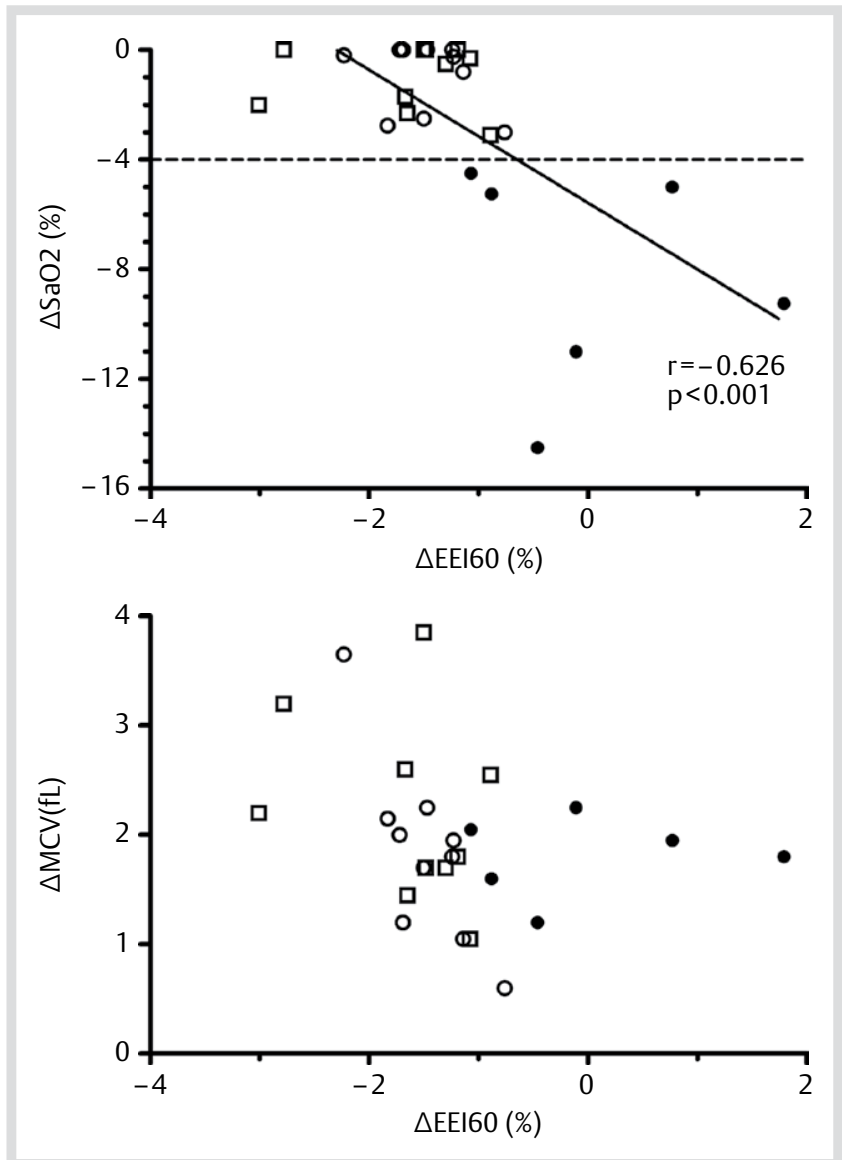

Fig. 2 Correlation plot between the increment of erythrocyte elongation index at $60 \mathrm{~Pa}\left(\triangle \mathrm{EEI} I 60=\mathrm{EEI} 60_{\text {post-exercise }}-\mathrm{EEI} 60_{\text {pre-exercise }}\right)$ and the mean arterial desaturation at the 2 last minutes of the incremental test (up) and the increment in mean corpuscular volume (MCV, down); in the sedentary $(\square)$, the trained non-EAIH $(\odot)$ and the trained EIAH $(\bullet)$ subjects.

EIAH subjects showed the same exercise effects on lactate concentrations ( $\bullet$ Fig. 3) and MCV increment ( $\bullet$ Fig. 1) as the sedentary and the trained non-EIAH subjects. Therefore, the greater MCT-1 lactate transport activity that has been reported in these subjects [8] does not seem to have any effect on the erythrocyte cellular properties linked to ED.

Several studies have reported divergent results regarding the effect of exercise on MCV [3,28-30]. The exercise type (running vs. cycling), methodological issues, and more importantly exercise intensity, are important factors to take into account when MCV values are compared. However, none of the aforementioned studies reported data about exercise intensity or blood lactate levels, which are crucial for understanding the effects of exercise on MCV.

Our data reveal that RBC membrane-cytoskeleton properties in the trained-EIAH subjects must be affected distinctly. The erythrocyte bilayer membrane is connected to the cytoskeleton through interactions between the cytoplasmic domain of integral membrane proteins and the spectrin network. These linkages stabilizes the bilayer membrane and are organized into 2 structures: one around the ankyrin protein that links band 3 $\left(\mathrm{HCO}_{3}{ }^{-} / \mathrm{Cl}^{-}\right.$exchanger, SLC4A1) and $\beta$-spectrin [18], and the other around protein $4.1 \mathrm{R}$ that forms a junctional complex with actin and $\beta$-spectrin, and is linked to several membrane proteins, which are probably organized in a band 3-centred metabolon 


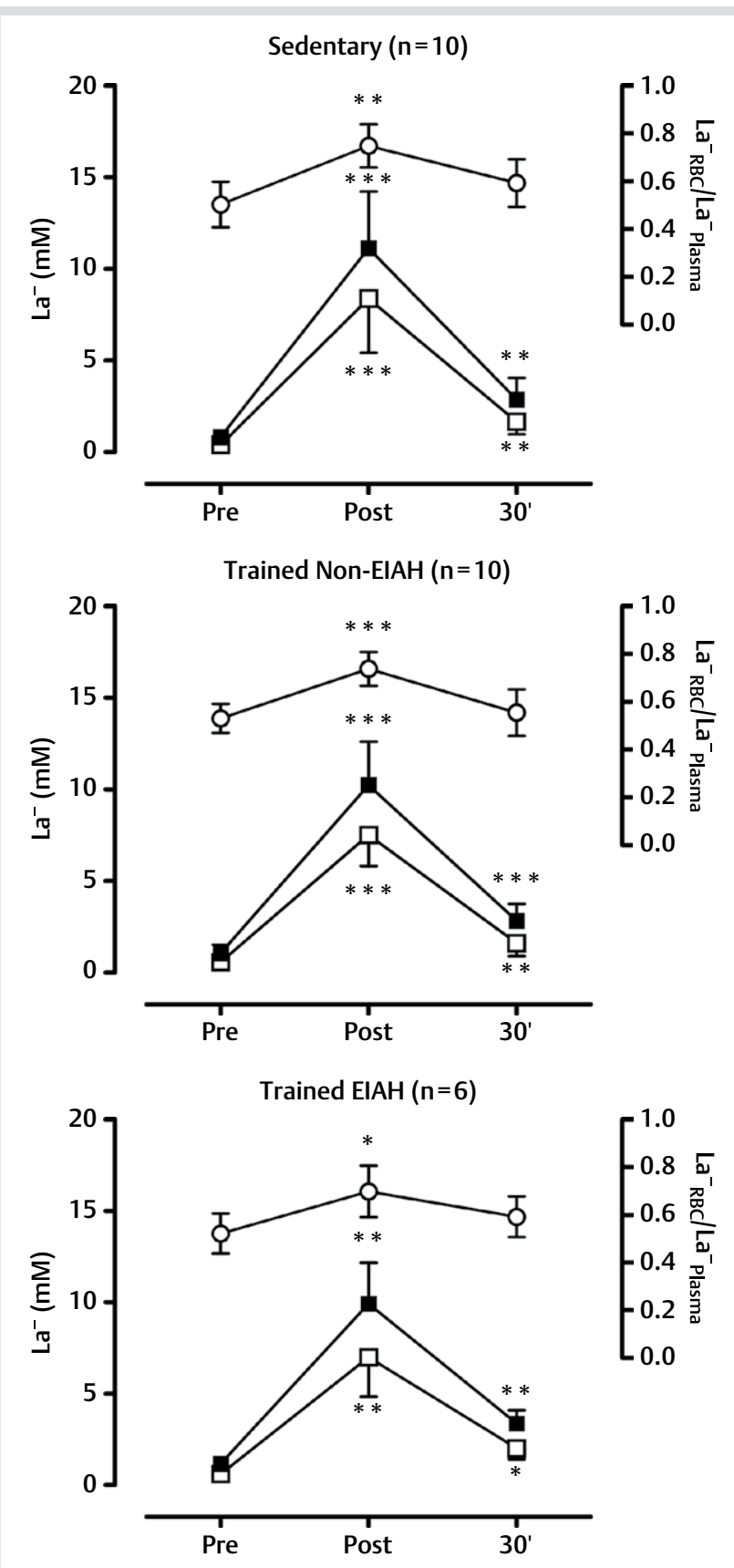

Fig. 3 Effect of exercise and the 30-min recovery period on lactate concentrations in plasma ( $\square$ ), inside erythrocyte $(\square)$ and on the insideto-outside erythrocyte lactate ratio $(O)$ in the sedentary, the trained subjects without exercise-induced arterial hypoxemia (EIAH) and the trained subjects with EIAH. Symbols represent the mean values and error bars denote standard deviation. ${ }^{*} \mathrm{p}<0.05,{ }^{*}{ }^{*} \mathrm{p}<0.01,{ }^{*}{ }^{*}{ }^{*} \mathrm{p}<0.001$ vs. pre-exercise.

[24]. Weakening these interactions leads to decreased membrane stability and enhanced ED $[16,17]$. Interestingly, it has been recently shown that deoxyhemoglobin binds to the cytoplasmic domain of band 3 inhibiting the ankyrin-band 3 interactions [27]. Therefore, the lowered $\mathrm{O}_{2}$ saturation during exercise in the trained-EIAH subjects can be the factor that explains the lack of exercise-induced effects on ED, if compared to sedentary and non-EIAH trained subjects, and despite having the same impact on lactate concentration, MCV and MCHC.
It has been previously hypothesized that ED can play a role in the appearance of EIAH [7]. Yet according to our results and current knowledge on RBC membrane physiology, it rather seems that the appearance of EIAH should have an effect on ED. In line with this, drops in $\mathrm{SaO}_{2}$ should improve ED at high exercise intensity. This would prove to be an adaptive mechanism for improving pulmonary and muscular perfusion, and thus allowing stabilization of $\mathrm{SaO}_{2}$ and muscular metabolism. It could, therefore, explain why high exercise intensity can be achieved despite impaired $\mathrm{O}_{2}$ arterial transport in the EIAH subjects. In fact, no differences in maximal $\mathrm{O}_{2}$ consumption or higher lactate concentrations were found when comparing trained EIAH and non-EIAH, although in the former, the anaerobic threshold was found at a lower $\mathrm{VO}_{2}$ max percentage and the maximal RER was higher.

In conclusion, ED is not impaired by intense exercise when $\mathrm{SaO}_{2}$ considerably drops. This may be related to a newly discovered $\mathrm{O}_{2}$-driven cellular mechanism that regulates membranecytoskeleton interactions [27], which may constitute an adaptive mechanism in exercise.

\section{Acknowledgements}

$\nabla$

This research has been supported by grants DEP2012-37494 from the Spanish Goverment (to MR) and grant 2013-168-001 from Catholic University of Valencia (to JRB). RA is a predoctoral fellow of Catholic University of Valencia.

Conflict of interest: None of the authors have any conflict of interest.

\footnotetext{
Affiliations

${ }^{1}$ Research Universitary Institute Dr. Viña Giner, Molecular and Mitochondria Medicine, Catholic University of Valencia San Vicente Mártir, Valencia, Spain ${ }^{2}$ Department of Physiology, University of Valencia, Valencia, Spain

${ }^{3}$ Fundación Investigación Hospital Clínico Universitario/INCLIVA, Valencia, Spain

${ }^{4}$ Department of Biomedical Sciences for Health, Università degli Studi di Milano, Milano, Italy

${ }^{5}$ Faculty of Medicine, Catholic University of Valencia “San Vicente Mártir”,

Valencia, Spain

${ }^{6}$ Department of Physical Education and Sports, University of Valencia,

Valencia, Spain
}

\section{References}

1 Aguilaniu B, Flore P, Perrault H, Page JE, Payan E, Lacour JR. Exerciseinduced hypoxaemia in master athletes: effects of a polyunsaturated fatty acid diet. Eur J Appl Physiol 1995; 72: 44-50

2 Aguilaniu B, Flore P, Page E, Maitre J, Lacour JR, Perrault H. Effects of indomethacin and polyunsaturated fatty acid diet on exercise-induced hypoxaemia in master athletes. Eur J Appl Physiol 1998; 77: 81-88

3 Aguilo A, Castano E, Tauler P, Guix MP, Serra N, Pons A. Participation of blood cells in the changes of blood amino acid concentrations during maximal exercise. J Nutr Biochem 2000; 11: 81-86

4 Baskurt OK, Meiselman HJ. Blood Rheology and Hemodynamics. Semin Thromb Hemost 2003; 29: 435-450

5 Baskurt OK, Boynard M, Cokelet GC, Connes P, Cooke BM, Forconi S, Liao F, Hardeman MR, Jung F, Meiselman HJ, Nash G, Nemeth N, Neu $B$, Sandhagen B, Shin S, Thurston G, Wautier JL. New guidelines for hemorheological laboratory techniques. Clin Hemorheol Microcirc 2009; 42: 75-97

6 Betticher DC, Reinhart WH, Geiser J. Effect of RBC shape and deformability on pulmonary $\mathrm{O} 2$ diffusing capacity and resistance to flow in rabbit lungs. J Appl Physiol 1995; 78: 778-783

7 Brun J-F, Varlet-Marie E, Connes P, Aloulou I. Hemorheological alterations related to training and overtraining. Biorheology 2010; 47: 95-115 
8 Connes P, Bouix D, Py G, Caillaud C, Kippelen P, Brun J-F, Varray A, Prefaut $C$, Mercier J. Does exercise-induced hypoxemia modify lactate influx into erythrocytes and hemorheological parameters in athletes? J Appl Physiol 2004; 97: 1053-1058

9 Connes P, Bouix D, Py G, Prefaut C, Mercier J, Brun J-F, Caillaud C. Opposite effects of in vitro lactate on erythrocyte deformability in athletes and untrained subjects. Clin Hemorheol Microcirc 2004; 31: 311-318

10 Connes P, Simmonds MJ, Brun JF, Baskurt OK. Exercise hemorheology: classical data, recent findings and unresolved issues. Clin Hemorheol Microcirc 2013; 53: 187-199

11 Connes P, Bouix D, Durand F, Kippelen P, Mercier J, Prefaut C, Brun JF, Caillaud $C$. Is hemoglobin desaturation related to blood viscosity in athletes during exercise? Int J Sports Med 2004; 25: 569-574

12 Evans E, Mohandas N, Leung A. Static and dynamic rigidities of normal and sickle erythrocytes. Major influence of cell hemoglobin concentration. J Clin Invest 1984; 73: 477-488

13 Galy O, Hue O, Boussana A, Peyreigne C, Mercier J, Préfaut C. Blood rheological responses to running and cycling: a potential effect on the arterial hypoxemia of highly trained athletes? Int J Sports Med 2005; 26: 9-15

14 Harriss DJ, Atkinson G. Ethical standards in sport and exercise science research: 2014 update. Int J Sports Med 2013; 34: 1025-1028

15 Hildebrand A, Lormes W, Emmert J, Liu Y, Lehmann M, Steinacker JM. Lactate Concentration in Plasma and Red Blood Cells During Incremental Exercise. Int J Sports Med 2000; 21: 463-468

16 Manno S, Takakuwa Y, Nagao K, Mohandas N. Modulation of erythrocyte membrane mechanical function by beta-spectrin phosphorylation and dephosphorylation. J Biol Chem 1995; 270: 5659-5665

17 Manno S, Takakuwa Y, Mohandas N. Modulation of erythrocyte membrane mechanical function by protein 4.1 phosphorylation. J Biol Chem 2005; 280: 7581-7587

18 Mohandas N, Gallagher PG. Red cell membrane: past, present, and future. Blood 2008; 112: 3939-3948

19 Nielsen HB. Arterial desaturation during exercise in man: implication for 02 uptake and work capacity. Scand J Med Sci Sports 2003; 13 : 339-358

20 Nihill MR, McNamara DG, Vick RL. The effects of increased blood viscosity on pulmonary vascular resistance. Am Heart J 1976; 92: 65-72
21 Prefaut C, Durand F, Mucci P, Caillaud C. Exercise-induced arterial hypoxaemia in athletes: a review. Sports Med 2000; 30: 47-61

22 Romagnoli $M$, Alis R, Martinez-Bello V, Sanchis-Gomar F, Aranda R, Gomez-Cabrera MC. Blood rheology effect of submaximal exercise on young subjects. Clin Hemorheol Microcirc 2014; 56: 111-117

23 Ruef P, Pöschl JMB, Linderkamp O, Schmid-Schönbein $H$. The shear stress diffractometer Rheodyn SSD for determination of erythrocyte deformability II. Sensitivity to detect abnormal erythrocyte deformability. Clin Hemorheol Microcirc 1996; 16: 749-752

24 Salomao M, Zhang X, Yang Y, Lee S, Hartwig JH, Chasis JA, Mohandas $N$, An X. Protein 4.1R-dependent multiprotein complex: new insights into the structural organization of the red blood cell membrane. PNAS 2008; 105: 8026-8031

25 Schmid-Schönbein H, Ruef $P$, Linderkamp 0 . The shear stress diffractometer Rheodyn SSD for determination of erythrocyte deformability I. Principles of operation and reproducibility. Clin Hemorheol Microcirc 1996; 16: 745-748

26 Smith JA, Telford RD, Kolbuch-Braddon $M$, Weidemann MJ. Lactate/H+ uptake by red blood cells during exercise alters their physical properties. Eur J Appl Physiol 1997; 75: 54-61

27 Stefanovic M, Puchulu-Campanella E, Kodippili G, Low PS. Oxygen regulates the band 3-ankyrin bridge in the human erythrocyte membrane. Biochem J 2013; 449: 143-150

28 Sureda A, Tauler P, Aguilo A, Fuentespina E, Cordova A, Tur JA, Pons A. Blood cell NO synthesis in response to exercise. Nitric Oxide 2006; 15: $5-12$

29 Tauler P, Aguilo A, Gimeno I, Fuentespina E, Tur JA, Pons A. Influence of vitamin $C$ diet supplementation on endogenous antioxidant defences during exhaustive exercise. Pflugers Arch 2003; 446: 658-664

30 Tauler P, Aguilo A, Guix P, Jimenez F, Villa G, Tur JA, Cordova A, Pons A. Pre-exercise antioxidant enzyme activities determine the antioxidant enzyme erythrocyte response to exercise. J Sports Sci 2005; 23: 5-13

31 Vent-Schmidt J, Waltz X, Pichon A, Hardy-Dessources MD, Romana M, Connes $P$. Indirect viscosimetric method is less accurate than ektacytometry for the measurement of red blood cell deformability. Clin Hemorheol Microcirc 2013 